\title{
Incidental Chronic Lymphocytic Leukemia Diagnosed Following Radical Prostatectomy for Prostate Cancer : a Case Report
}

\section{Ghassen Tlili}

Sahloul University Hospital: Hopital Sahloul https://orcid.org/0000-0003-1709-9900

houssem ammar ( $\nabla$ drhoussemammar@gmail.com )

Universite de Sousse Faculte de Medecine de Sousse

wiem majdoub

Universite de Sousse Faculte de Medecine de Sousse

sonia dziri

Universite de Sousse Faculte de Medecine de Sousse

waad farhat

Universite de Sousse Faculte de Medecine de Sousse

emir akacha

Universite de Sousse Faculte de Medecine de Sousse

rahul gupta

synergy institute of medical sciences

khaled ben ahmed

Universite de Sousse Faculte de Medecine de Sousse

awatef azzabi

Universite de Sousse Faculte de Medecine de Sousse

mehdi jaidane

Universite de Sousse Faculte de Medecine de Sousse

\section{Case report}

Keywords: Radical prostatectomy, Chronic lymphocytic leukemia, Prostate carcinoma

Posted Date: May 5th, 2021

DOI: https://doi.org/10.21203/rs.3.rs-476854/v1

License: (c) (i) This work is licensed under a Creative Commons Attribution 4.0 International License.

Read Full License 


\section{Abstract}

Background: chronic lymphocytic leukemia (CLL) patients have a high risk of occurrence of secondary cancers. This risk is three times higher for all cancers and eight times higher for skin cancer. The coexistence of CLL and adenocarcinoma of the prostate is rare.

Case presentation: We report a case of 66-year-old man who underwent radical prostatectomy for prostate carcinoma. The final histopathological diagnosis of Gleason 7 adenocarcinoma of the prostate with incidental chronic lymphocytic leukemia (CLL) was made. No further investigations or treatment was offered due to the age, low grade and the stage of the disease. At last follow-up of 12 months, the patient is alive, without disease progression for both lymphoma and prostate with PSA value of $0.1 \mathrm{ng} / \mathrm{ml}$.

Conclusion: Early detection of lymphoma after radical prostatectomy will allow optimal management. The analysis of this link requires therefore additional investigations.

\section{Background}

Radical prostatectomy is the gold standard for treatment of prostate cancer [1]. The detection of lymph node before surgery is difficult, because it is frequently microscopic and therefore undetectable using existing imaging modalities[1-3]. The chronic lymphocytic leukemia (CLL) patients, have a high risk of developing a secondary cancers, however the coexistence of CLL and prostate adenocarcinoma is rare [3, 4]. We report a case of Incidental chronic lymphocytic leukemia(CLL) diagnosed following radical prostatectomy for prostate adenocarcinoma of the prostate with concomitant chronic lymphocytic leukemia, in 66-year-old man .

\section{Case Description}

A 66-year-old man presented with history of lower urinary tract symptoms for 4 months .He rapported nocturia, Weak urine stream and an urgent need to urinate. Abdominal examination was unremarkable, and digital rectal examination revealed a prostatic hypertrophy. Laboratory tests showed high Prostate specific antigen (PSA) level $(5.06 \mathrm{ng} / \mathrm{ml})$. Liver and renal function tests were normal. Abdominal magnetic resonance imaging (MRI) showed the presence of a posterior prostate lesion measuring $16 \mathrm{~mm}$, located in the left peripheral zone. The lesion was hypointense on T2-weighted (T2W) and on apparent diffusion coefficient (ADC) images, however it was hyperintense on Diffusion-weighted imaging (DWI images) (Fig. 1). Based on these findings, the prostate lesion was classified as category 5 according to PI-RADS (Prostate Imaging-Reporting and Data System) assessment score .MRI guided prostate biopsy confirmed the presence of Gleason $4+3=7$ adenocarcinoma of the prostate in 9 cores among 12 cores with a maximum core involvement of $90 \%$. A bone scan did not reveal metastatic bone disease. Based on those findings, a radical prostatectomy was done(bilateral wide local excision with bladder neck preservation and pelvic lymph node dissection).

On histopathology, bilateral, multifocal microacinar type of prostatic adenocarcinoma was identified. 
the resection margin was free from tumor cells. Lymph nodes and seminal gland examination showed a diffuse infiltration of small B-cells lymphocytes. A final Gleason score was $4+3=7$. On immunohistochemistry, the lymphoid infiltration positively stained for the CD20, CD5 and CD23 and negatively staining for CD3, CD10, cyclin D1 and Bcl 6(Fig. 2). Thus, the final histopathological diagnosis of Gleason 7 adenocarcinoma of the prostate, staged pT2c NO MX, associated to an incidental chronic lymphocytic leukemia (CLL), was made. The patient was then referred for hematological evaluation. No further investigations or treatment was offered due to the age, low grade and the stage of the disease. At last follow-up of 12 months, the patient is alive, without disease progression and the PSA value is normal.

\section{Discussion}

CLL is a lymphoproliferative syndrome characterized by a medullary proliferation of a clone B cell, which invade blood and lymphoid organs [5]. CLL patients have a high risk of association with adenocarcinomas. It is more likely to coincide with adenocarcinoma of the gastrointestinal tract, skin and breast, however it s coexistence with prastatic cancer is rare $[2,6]$. This association rate was $0,8 \%$ in Terris et al study including 1092 patients, who underwent radical prostatectomy, however this rate was $0 \%$ in Eisemberger et al study including 4319 patients [7].Tsimberidou et al reported a serie of 2028 patients diagnosed with CLL and small lymphocytic lymphoma, among which 551 patients had a history of other malignancies or developed other cancer during the follow-up period [8].

CLL patients have a high risk of occurrence of secondary cancers, which can reach 10-11 times higher than the normal population $[4,6]$. Some authors attribute this risk to the chemotherapeutic treatment of the lymphoma, other related it to to a defect in the cellular immune mechanisms whereas some authors to chance alone [2].

Some authors reported independent factors predicting development of other cancers such as older age, male sex, $\beta 2$-microglobulin $>3 \mathrm{mg} / \mathrm{L}$, Lactate dehydrogenase $>618 \mathrm{U} / \mathrm{L}$, Creatinine $>1.6 \mathrm{mg} / \mathrm{dL}$ [8]. However, author studies showed that patients with $17 p$ deletion, $6 q$ deletion, or $11 q$ deletion and/or trisomy 12 had an increased risk of developing other cancers compared with other patients [9].

Litterature review showed a good prognosis of CLL diagnosed after radical prostatectomy $[1,7]$. Due to the risk of developing prostate cancer in patients diagnosed with LLC, some author reported the need of prostatic cancer screening using PSA test [6].

\section{Conclusion}

Incidental chronic lymphocytic leukemia diagnosed following radical prostatectomy for prostate cancer is a rare situation with few cases reported in the literature. However many Risk factors have been reported, the origin of this accociation is still debated and analysis of this link requires therefore additional investigations. 


\section{Declarations}

\section{Ethical approval:}

All procedures performed in studies involving human participants were in accordance with the ethical standards of the institutional and/or national research committee and with the 1964 Helsinki declaration and its later amendments or comparable ethical standards. approval references approved by Sahloul hospital ethic committee: U2341

\section{Acknowledgment:}

None

\section{Financial support:}

None

\section{Informed consent:}

The patient provided informed written consent prior to submission of this manuscript.

\section{Conflict of interest:}

The authors declare no conflict of interest.

\section{Author contributions:}

Wiem Majdoub- Editing of manuscript, data collection, Anatomopathology analysis

Ghassen tlili - Editing of manuscript, supervision of the manuscript

Houssem Ammar- Editing of manuscript, literature review, drafting the manuscript

Emir akacha- Editing of manuscript, data collection

Sonia Dziri- Data collection, Editing of the manuscript

Waad Farhat- data collection, Editing of manuscript

Mehdi jaidane- Editing of manuscript, data collection

Rahul Gupta- Editing of manuscript, literature review, drafting the manuscript

Khaled ben ahmed - manuscript correction, supervision of the manuscript

Awatef Azzabi - Supervision of the manuscript, manuscript correction 


\section{References}

1. El-Taji O, Omer A, Al-Mitwalli A, Agarwal S et al . Incidental Lymphoplasmacytic Lymphoma Diagnosed Following Robotic-Assisted Laparoscopic Prostatectomy for Prostate Cancer. Curr Urol 2019;13:166-168.

2. R Gros, S Richter, L Bechar .Prostatic carcinoma with concomitant non-Hodgkin lymphoma. Report of 2 cases. Urol Int. 1984;39(2):121-2.

3. Fournier G, Valeri A, Mangin P, Cussenot O. Cancer de la prostate Épidémiologie. Facteurs de risques. Anatomopathologie. Ann Urol 2004;38:187-206.

4. Ather $\mathrm{MH}$, Memon A. Acute urinary retention: a primary manifestation of chronic lymphocytic leukemia and organ confined prostate cancer. J Pak Med Assoc 1999;49:124-5.

5. Binet JL, Caligaris-Cappio F, Catovsky D, Cheson B, Davis T, Dighiero G, et al. Perspectives on the use of new diagnostic tools in the treatment of chronic lymphocytic leukemia. Blood 2006; 107:859-61.

6. Hatim Nafil, Illias Tazi, L. Mahmal. Cancer de la prostate et leucémie lymphoïde chronique. African Journal of Urology 19(1):35-38

7. Ballario R, Beltrami P, Cavalleri S, Ruggera $L$ et al . An unusual pathological finding of chronic lymphocytic leukemia and adenocarcinoma of the prostate after transurethral resection for complete urinary retention: case report. BMC 2004;4:95.

8. Tsimberidou AM, Wen S, McLaughlin P, O’Brien S et al. Other malignancies in chronic lymphocytic leukemia/small lymphocytic lymphoma. J Clin Oncol 2009; 27: 904-10.

9. Tsimberidou AM, Wen S, O'Brien S, McLaughlin P et al.Assessment of chronic lymphocytic leukemia and small lymphocytic lymphoma by absolute lymphocyte counts in 2,126 patients: 20 years of experience at the University of Texas M.D. Anderson Cancer Center. J Clin Oncol 2007;25(29):464856.

\section{Figures}




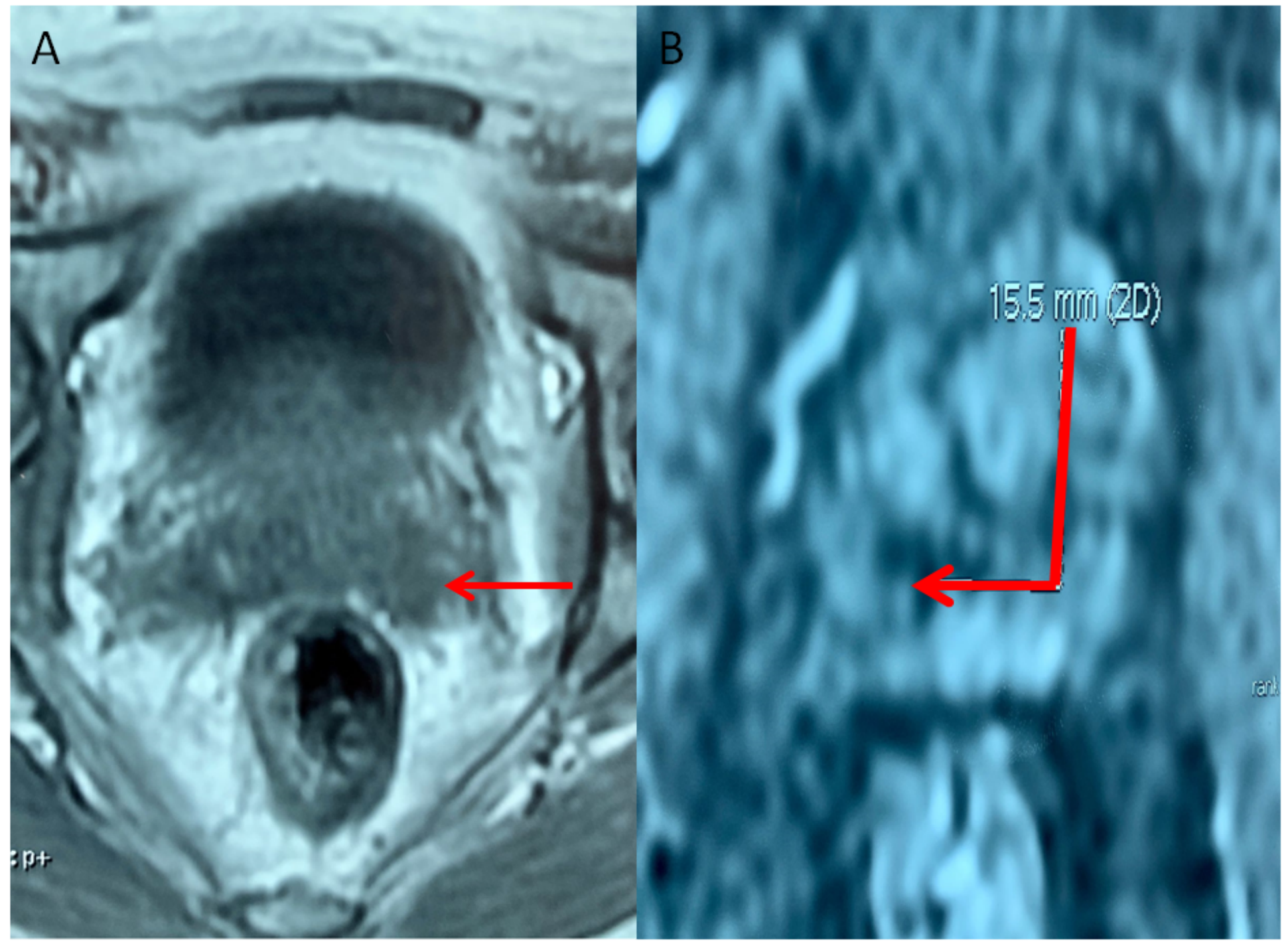

\section{Figure 1}

Abdominal magnetic resonance imaging (MRI) showed the presence of a posterior prostate lesion measuring $16 \mathrm{~mm}$, located in the left peripheral zone Magnetic hypointense on T2W (A) and markedly hyperintense on DWI (B).

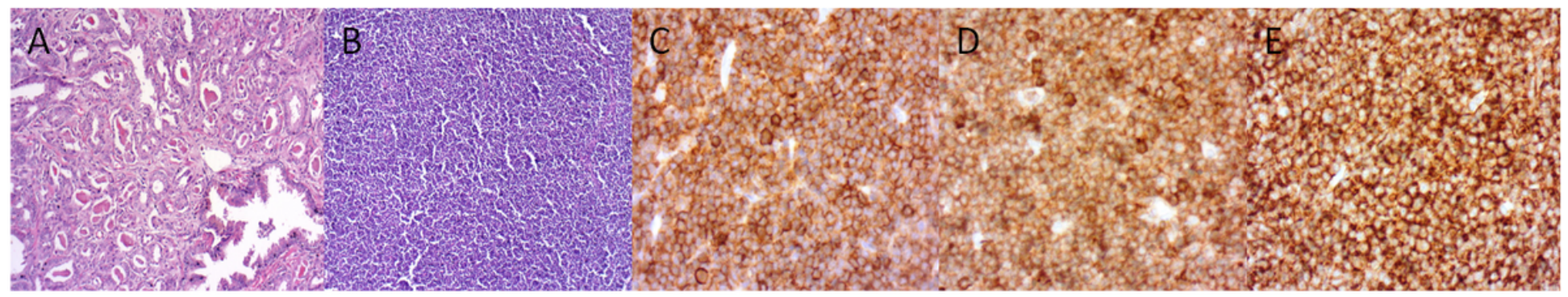

Figure 2 
Microscopic examination of the prostate tissue showing crowded glands of adenocarcinoma with dense eosinophilic crystalloids [Gleason score $7(4+3)$ ] (A). The pelvic lymph node examination revealed small, round tumor cells with low grade of differentiation (H\&E) (B) and positive staining for CD20 (C), CD5 (D) and $\mathrm{CD} 23(\mathrm{E})$. 